\title{
Abdominal decompression plays a major role in early postparacentesis haemodynamic changes in cirrhotic patients with tense ascites
}

\author{
J Cabrera, L Falcón, E Gorriz, M D Pardo, R Granados, A Quinones, M Maynar
}

Hepatology Section, Unit of Investigation, Hospital General Universitario de Gran Canaria, Doctor Negrín, Universidad de Las Palmas de Gran Canaria, Spain

J Cabrera

L Falcón

R Granados

A Quinones

Interventional Vascular Radiology Section, Unit of Investigation, Hospital General Universitario de Gran Canaria, Doctor Negrín, Universidad de Las Palmas de Gran Canaria, Spain E Gorriz

M D Pardo

M Maynar

Correspondence to: Dr J Cabrera, Unidad de Investigación del Hospital General Universitario de Gran Canaria, Doctor

Negrín, Las Palmas de Gran Canaria, Spain.

med004083@nacom.es

Accepted for publication 8 August 2000

\begin{abstract}
Background-Some cirrhotic patients with tense ascites who undergo paracentesis develop a circulatory dysfunction syndrome, manifested by an increase in plasma renin activity. Recently, a significant inverse correlation between postparacentesis changes in plasma renin activity and systemic vascular resistance has been demonstrated in these patients, suggesting that peripheral arterial vasodilatation could be responsible for this circulatory dysfunction, but the mechanisms by which tense ascites removal induces such changes are unknown

Aim-To investigate the role of a decrease in intra-abdominal pressure (IAP) in the development of early postparacentesis haemodynamic changes

Methods-Eleven cirrhotic patients with tense ascites received a large volume paracentesis. A specially designed pneumatic girdle was used to compress the abdomen to avoid a decrease in IAP during ascites removal. Haemodynamic studies were performed before paracentesis, one hour after ascites flow stopped, and 30 minutes after pneumatic girdle deflation

Results-When IAP was maintained at its original level, no haemodynamic changes were observed, despite large volume paracentesis. However, a significant decrease in systemic vascular resistance was seen immediately after pneumatic girdle deflation

Conclusions-Early haemodynamic changes after paracentesis are avoided if IAP is maintained at its original level. The abrupt decrease in IAP could be the trigger for the development of the initial haemodynamic changes that eventually produce postparacentesis circulatory dysfunction.

(Gut 2001;48:384-389)
\end{abstract}

Keywords: cirrhosis; ascites; postparacentesis circulatory dysfunction; haemodynamic changes; intra-abdominal pressure

Previous studies have shown that large volume paracentesis in cirrhotic patients with tense ascites is followed by circulatory changes which in some cases can be associated with activation of the renin-angiotensin-aldosterone system. ${ }^{1-3}$ Few of these patients show serious clinical complications in the short term, but patients in whom plasma renin activity (PRA) increases to greater than $50 \%$ over basal levels have a tendency to suffer clinical consequences, such as faster ascites reaccumulation due to an increase in renal sodium retention, hyponatraemia, and functional renal failure. ${ }^{1}$ Moreover, the importance of this disturbance was highlighted by a long term follow up study in which patients developing this level of vasoactive activation had reduced survival. ${ }^{4}$ However, other studies have failed to demonstrate any disturbances in systemic and renal haemodynamics 48 hours after a single 5 litre paracentesis. ${ }^{5}$

The mechanisms by which massive paracentesis can induce activation of the vasoactive system in some cirrhotic patients are unknown. It has been shown that simultaneous intravenous administration of plasma expanders during paracentesis minimises activation of the vasoactive systems, suggesting that hypovolaemia could be responsible for the postparacentesis circulatory dysfunction (PCD). ${ }^{13467}$ But several studies have failed to demonstrate a significant reduction in intravascular volume after ascites removal. ${ }^{8}$ Alternatively, an acute imbalance between vascular capacity and available plasma volume has been suggested as one of the factors responsible for PCD. $.^{11} \mathrm{Re}-$ cently, Ruiz del Arbol et al demonstrated a significant inverse correlation between changes in systemic vascular resistance (SVR) and PRA on day 6 after ascites removal. ${ }^{12}$ This finding strongly suggests that PCD could be caused by enhancement of peripheral arterial vasodilatation that characterises these patients. The mechanism by which paracentesis induces this arteriolar vasodilatation and the vascular area where these changes occur have not been identified. One of the striking consequences of paracentesis is an early and abrupt decrease in intra-abdominal pressure (IAP), occurring simultaneously with changes in systemic haemodynamics. ${ }^{313}$ The aim of our study was to investigate the possible role of abdominal decompression on the initiation of postparacentesis haemodynamic changes.

Abbreviations used in this paper: PRA, plasma renin activity; FHVP, free hepatic vein pressure; WHVP, wedge hepatic vein pressure; HVPG, hepatic venous pressure gradient; IAP, intra-abdominal pressure; $\mathrm{CO}$, cardiac output; MAP, mean arterial pressure; RAP, right atrial pressure; SVR, systemic vascular resistance; PCD, postparacentesis circulatory dysfunction. 


\section{Patients and methods}

STUDY POPULATION

The study was performed in 11 cirrhotic patients admitted to our unit for an episode of tense ascites. Diagnosis of cirrhosis was based on history, physical examination, laboratory data, endoscopic evidence of oesophageal varices, ultrasonography data, and/or liver biopsy. $\mathrm{Pa}-$ tients were considered for the study if they fulfilled the following criteria: (1) absence of clinical, biochemical, or echographic data suggesting hepatocellular carcinoma; (2) absence of hepatic encephalopathy, gastrointestinal haemorrhage, or severe systemic infection in the past four weeks; (3) serum bilirubin $<10 \mathrm{mg} / \mathrm{dl}$; (4) quick index $>40 \%$; (5) platelet count $>40000 / \mathrm{mm}^{3}$; and (6) serum creatinine <3 $\mathrm{mg} / \mathrm{dl}$. Patients with respiratory, cardiac, or renal diseases and those receiving vasoactive drugs were excluded. All patients gave written informed consent to participate in the study which was approved by the clinical investigation and ethics committee of our hospital.

\section{STUDY DESIGN}

After admission, diuretic treatment was withdrawn and a diet was provided containing 50 $\mathrm{mEq} /$ day of sodium without water restriction. On the fifth day, 24 hour urine was collected to measure electrolytes. On the morning of the sixth day, fasting blood samples were obtained to measure serum electrolytes, and standard kidney and liver function. Ascitic fluid was sampled for leucocyte count. Patients were then transferred to the haemodynamic laboratory where a basal haemodynamic study (see below) was performed. Before starting paracentesis, a $25 \mathrm{~cm}$ wide pneumatic girdle, specially designed for this study, was adjusted around the abdominal perimeter. The girdle consisted of a non-elastic adjustable sheath covering two pneumatic balloons.

Inflation was by means of a cuff to maintain IAP $1-2 \mathrm{~mm} \mathrm{Hg}$ above the basal level during ascites removal. Under sterile conditions, using the Seldinger technique, a three lumen central venous 12 French gauge catheter (Ref CS12123-E; Arrow International Inc, Bernville Road, Pennsylvania 19605, USA) was inserted into the abdominal cavity through a lateral hole located in the left side of the girdle. One of the catheters was connected to a digital pressure monitor to obtain a continuous recording of IAP. The others were connected to a graduated collecting plastic bag. One hour after ascites flow through the paracentesis catheter had stopped, a second haemodynamic study was performed while the pneumatic girdle was inflated. Following this haemodynamic study, the pneumatic girdle was completely deflated. In nine of 11 patients, right atrial pressure (RAP), cardiac output (CO), and mean arterial pressure (MAP) were measured five minutes after girdle deflation and SVR calculated (these haemodynamic measurements were added to the protocol after study of the two first patients). In all patients, a complete haemodynamic study was again performed 30 minutes after girdle deflation. Human albumin ( $8 \mathrm{~g}$ for each litre of ascitic fluid removed) was admin- istered intravenously over 60-80 minutes following the last haemodynamic measurement, to avoid the risk of developing PCD.

\section{HAEMODYNAMIC MEASUREMENTS}

Under local anaesthesia and using the Seldinger technique, an angiographic 7 French gauge balloon tipped catheter (Cordis Company, Miami, Florida, USA) was inserted into the right femoral vein and advanced into the main right hepatic vein under fluoroscopic guidance to measure wedge hepatic vein pressure (WHVP) and free hepatic vein pressure (FHVP). The occluded position of the catheter was checked by the absence of reflux after injection of $2 \mathrm{ml}$ of contrast medium. Through the right internal jugular vein, a Swan-Ganz thermodilution catheter (Model 744H-7.5F; Baxter Healthcare Corporation, Edwards Critical-Care Division, Irvine California, USA) was advanced into the main pulmonary artery for continuous monitoring of RAP and CO. CO was measured with the monitor Vigilance (Baxter Healthcare Corporation). The mean value of the three last measurements was used as the final value. MAP was recorded continuously using an angiographic catheter (Multipurpose 5F; Cordis Company, Miami, Florida, USA) advanced into the abdominal aorta using the Seldinger technique, through the right femoral artery. All pressures were measured using a high sensitive pressure transducer (Minimón 7132-A; Kontron Medical International, Bernerstrasse Sud, Zurich, Switzerland) calibrated before each measurement. The axillary midline level was considered the zero reference point. The mean of three measurements was used as the final value for all haemodynamic parameters. SVR (dyns $/ \mathrm{cm}^{5}$ ) was calculated as $((\mathrm{MAP}-\mathrm{RAP}) \times 80) / \mathrm{CO}$. Throughout the study ECG and oxygen saturation were continuously monitored.

\section{STATISTICAL ANALYSIS}

Statistical analysis was performed using the ANOVA test for repetitive measurements and the Newman-Keuls test for hypothesis testing. Previously, the Komolgorov-Smirnov test was used to ensure that the parameters followed a normal distribution. The R-Sigma statistical package (Horus Hardware SA, Madrid, Spain) was used. Results are reported as mean (SD). Significance was established at $\mathrm{p}<0.05$.

\section{Results}

\section{CLINICAL CHARACTERISTICS OF PATIENTS}

Eleven patients (all men) were included in the study (table 1). Most had advanced cirrhosis, as indicated by a Child-Pugh score of 9.1 (1). Mean serum sodium concentration was 133 (5.6) $\mathrm{mEq} / \mathrm{l}$ (range 121-140). Two patients had hyponatraemia, arbitrarily defined as a serum sodium concentration less than 130 $\mathrm{mEq} / \mathrm{l}$. All patients had urinary sodium excretion less than $10 \mathrm{mEq} / 24$ hours (mean 5.2 (0.4); range 0-9.6 mEq/24 hours). Peripheral oedema was present in seven patients. Nine patients had previous episodes of ascites. The mean volume of ascites removed was 8600 
Table 1 Clinical and biochemical characteristics of the patients included in the study

\begin{tabular}{|c|c|c|c|c|c|c|c|c|c|c|}
\hline $\begin{array}{l}\text { Patient } \\
\text { No }\end{array}$ & Aetiology & Age (y) & $\begin{array}{l}\text { Child-Pugh } \\
\text { score }\end{array}$ & $\begin{array}{l}\text { Serum } \\
\text { creatinine } \\
(\mu m o l / l)\end{array}$ & $\begin{array}{l}\text { Serum } \\
\text { sodium } \\
(\text { mmolll })\end{array}$ & $\begin{array}{l}\text { Serum } \\
\text { albumin } \\
(\mathrm{g} / \mathrm{l})\end{array}$ & $\begin{array}{l}\text { Serum } \\
\text { bilirubin } \\
(\mu m o l / l)\end{array}$ & $\begin{array}{l}\text { Quick } \\
\text { index } \\
(\%)\end{array}$ & $\begin{array}{l}\text { Sodium } \\
\text { excretion } \\
\text { (mmol/day) }\end{array}$ & $\begin{array}{l}\text { Volume of } \\
\text { paracentesis } \\
\text { (ml) }\end{array}$ \\
\hline 1 & ETOH & 54 & 9 & 84.864 & 140 & 28.9 & 47.88 & 60 & 7.8 & 6000 \\
\hline 2 & ETOH & 68 & 9 & 45.084 & 134 & 27 & 25.65 & 100 & 112.7 & 6000 \\
\hline 3 & ETOH & 45 & 8 & 82.212 & 137 & 33 & 25.65 & 92 & 2 & 12000 \\
\hline 4 & ETOH & 55 & 8 & 117.572 & 130 & 30 & 29.07 & 100 & 115.2 & 8500 \\
\hline 5 & VIRAL & 47 & 9 & 85.748 & 128 & 28 & 47.88 & 71 & 7.2 & 8500 \\
\hline 6 & ETOH & 65 & 9 & 55.692 & 137 & 21.1 & 18.81 & 80 & 82 & 14000 \\
\hline 7 & ETOH & 43 & 9 & 62.764 & 131 & 23.7 & 64.98 & 60 & 0 & 7000 \\
\hline 8 & ETOH & 59 & 11 & 91.936 & 121 & 20 & 78.66 & 100 & 0 & 5000 \\
\hline 9 & ETOH & 59 & 9 & 45.968 & 135 & 25 & 23.94 & 81 & 2 & 8000 \\
\hline 10 & ETOH & 52 & 8 & 83.096 & 140 & 30 & 32.49 & 74 & 9.1 & 10000 \\
\hline 11 & ETOH & 46 & 11 & 74.256 & 135 & 17.4 & 54.72 & 60 & 1.48 & 10000 \\
\hline Mean & & 53.9 & 9.09 & 75.381 & 133.454 & 25.827 & 40.884 & 79.8 & 30.861 & 8636 \\
\hline SD & & 8.3 & 1.0 & 21.653 & 5.645 & 4.840 & 19.353 & 16.3 & 47.355 & 2721 \\
\hline
\end{tabular}

ETOH, alcohol.
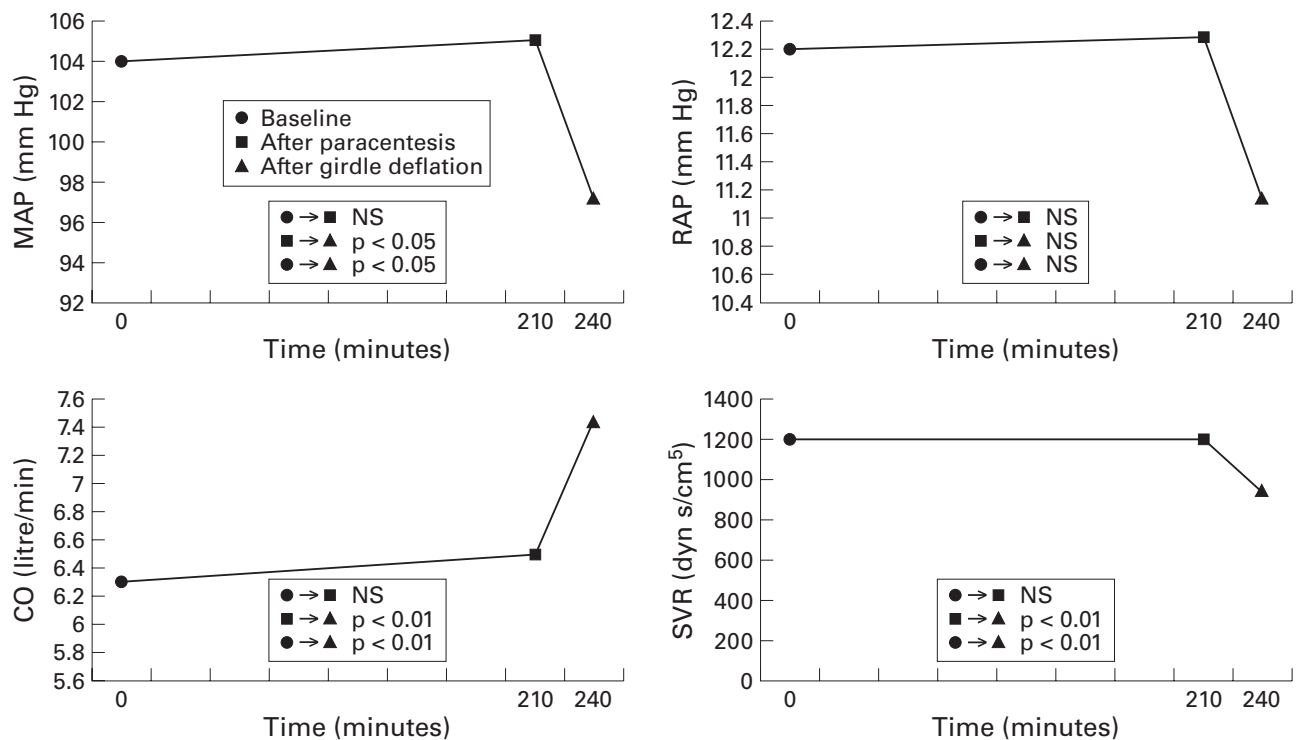

Figure 1 Systemic haemodynamic parameters at baseline, after completion of paracentesis, and 30 minutes after girdle deflation. MAP, mean arterial pressure right; $R A P$, right atrial pressure; $C O$, cardiac output; SVR, systemic vascular resistance.

(2700) $\mathrm{ml}$ (range 5000-14 $000 \mathrm{ml}$ ). Mean time for completion of paracentesis was 60 (20) minutes. No clinically evident side effects were observed during or after the study.

SYSTEMIC HAEMODYNAMIC DATA

Paracentesis did not provoke systemic haemodynamic changes while IAP was maintained constant. One hour after ascites removal, none of the haemodynamic parameters (CO, MAP, RAP, or SVR) was modified significantly. In contrast, 30 minutes after girdle deflation, a significant increase in CO (14.06\%) and a significant decrease in both MAP $(-7.71 \%)$ and SVR (-18.20\%) were observed (fig 1). RAP did not change significantly. Haemodynamic data are shown in table 2 . In nine patients in whom SVR was calculated five minutes after girdle deflation, a significant decrease in relation to the value postparacentesis was observed (1203.5 (254) v 1030 (218) dyn $\left.\mathrm{s} / \mathrm{cm}^{5} ; \mathrm{p}<0.01\right)$. In these patients a further significant decrease in SVR was observed 30 minutes after girdle deflation (944 (194) dyn s/ $\mathrm{cm}^{5}$;

Table 2 Haemodynamic parameters

\begin{tabular}{lccclll}
\hline & Basal & PP & \multicolumn{1}{l}{ PD } & Basal v PP & PP v PD & Basal v PD \\
\hline CO (1/min) & $6.3(1.5)$ & $6.5(1.4)$ & $7.4(1.5)$ & NS & $\mathrm{p}<0.01$ & $\mathrm{p}<0.01$ \\
RAP (mm Hg) & $12.2(6.1)$ & $12.3(6.7)$ & $11.1(6.6$ & $\mathrm{NS}$ & $\mathrm{NS}$ & $\mathrm{NS}$ \\
MAP (mm Hg) & $104(12.4)$ & $105(10.3)$ & $97.4(10.5)$ & $\mathrm{NS}$ & $\mathrm{p}<0.05$ & $\mathrm{p}<0.05$ \\
SVR (dyn s/cm $\left.{ }^{5}\right)$ & $1195(202)$ & $1175(236)$ & $953(175)$ & $\mathrm{NS}$ & $\mathrm{p}<0.01$ & $\mathrm{p}<0.01$ \\
FHVP $(\mathrm{mm} \mathrm{Hg})$ & $20.5(5.2)$ & $22.9(5.7)$ & $14.4(3.2)$ & $\mathrm{p}<0.05$ & $\mathrm{p}<0.01$ & $\mathrm{p}<0.01$ \\
WHVP (mm Hg) & $40.9(3.8)$ & $41.6(7.3)$ & $30.1(5.8)$ & $\mathrm{NS}$ & $\mathrm{p}<0.01$ & $\mathrm{p}<0.01$ \\
HVPG (mm Hg) & $20.3(6.2)$ & $18.7(4.7)$ & $15.6(4.5)$ & $\mathrm{p}<0.05$ & $\mathrm{NS}$ & $\mathrm{p}<0.01$ \\
IAP (mm Hg) & $23.1(6.4)$ & $24.9(7.0)$ & $10.3(3.3)$ & NS & $\mathrm{p}<0.01$ & $\mathrm{p}<0.01$ \\
\hline
\end{tabular}

Basal, values obtained at the baseline hamodynamic study; PP, values obtained at the postparacentesis haemodynamic study; PD, values obtained at the post-deflation haemodynamic study.

Basal $v$ PP, comparison between basal and postparacentesis values; PP $v$ PD, comparison between postparacentesis and post-deflation values; Basal $v \mathrm{PD}$, comparison between basal and post-deflation values.

Values are mean (SD).

CO, cardiac output; RAP, right atrial pressure; MAP, mean arterial pressure; SVR, systemic vascular resistance; FHVP, free hepatic vein pressure; WHVP, wedge hepatic vein pressure; HVPG, hepatic venous pressure gradient; IAP, intra-abdominal pressure. 
Table 3 Systemic vascular resistances (dyn $\mathrm{s} / \mathrm{cm}^{5}$ ), 5 and 30 minutes after girdle deflation (nine patients)

\begin{tabular}{llclc}
\hline Patient No & Basal & PP & 5 min PD & \multirow{2}{*}{30 min PD } \\
\hline 3 & 1288.71 & 1335.64 & 1233.8 & 1204.29 \\
4 & 1378.275 & 1364.96 & 1174 & 1060.74 \\
5 & 968.7875 & 911.8 & 746.32 & 748.537 \\
6 & 1420.444 & 1543.83 & 1248.4 & 1118.6 \\
7 & 1398.25 & 1331.67 & 1057.5 & 937.493 \\
8 & 947.3857 & 945.296 & 861.26 & 779.747 \\
9 & 1331.667 & 1438.2 & 1190.3 & 995.246 \\
10 & 875.5 & 851.109 & 665.83 & 608.762 \\
11 & 1343.773 & 1109.06 & 1092.8 & 1040.8 \\
Mean & 1216.98 & 1203.5 & 1030 & 943.8 \\
SD & 219.527 & 253.9 & 218.5 & 194.12
\end{tabular}

PP, postparacentesis; PD, post-deflation.

$\mathrm{p}<0.05)$. Individual data for the nine patients are shown in table 3.

SPLANCHNIC HAEMODYNAMIC DATA

IAP and WHVP remained unchanged one hour after paracentesis while the girdle was inflated but a moderate, significant increase in FHVP and hepatic venous pressure gradient (HVPG) were observed. Thirty minutes after girdle deflation, FHVP, WHVP, and IAP showed a parallel and significant decrease (fig 2). A slight but statistically significant decrease in HVPG was also observed. There was no correlation between the decrease in IAP and changes in CO or SVR after girdle deflation.

\section{Discussion}

Large volume paracentesis is an effective treatment for refractory ascites. The need for plasma volume expansion after paracentesis is controversial. Some studies have failed to demonstrate any disturbances in systemic or renal haemodynamics 48 hours after a single 5 litre paracentesis. ${ }^{514}$ However, other studies have highlighted the potential harmful effects on circulatory homeostasis after massive paracentesis in patients with tense ascites when performed without simultaneous plasma volume expansion. ${ }^{12}$ The pathogenesis of PCD remains incompletely understood. Recently,
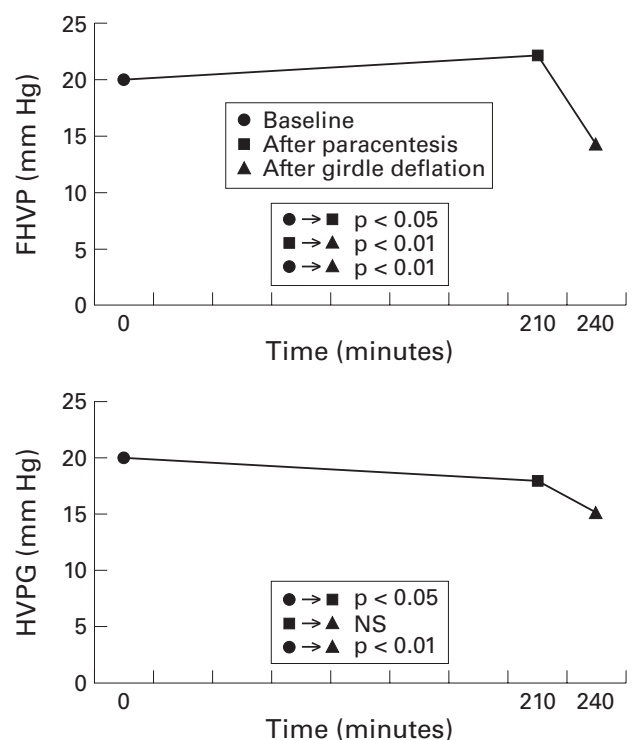

Ruiz del Arbol et al showed that the degree of activation of the renin-angiotensin system and sympathetic nervous system six days after paracentesis correlated inversely with changes in SVR. ${ }^{13}$ This finding suggests that accentuation of the peripheral arterial vasodilatation of cirrhosis occurs sometime after massive paracentesis. Similar results were observed in a recently published study ${ }^{14}$ showing that the increase in PRA and plasma aldosterone concentration, occurring days after massive paracentesis, is closely related to the decrease in SVR calculated by echo Doppler. Because cirrhotic patients have a widely dilated splanchnic vascular bed ${ }^{15}$ this would be the vascular area most susceptible to such changes.

Characteristic haemodynamic changes following paracentesis begin immediately after removal of the first decilitres of ascites; the greatest haemodynamic effect is seen after removal of $750 \mathrm{ml} .{ }^{13}$ Because an exponential decrease in abdominal pressure is a striking feature occurring during removal of tense ascites, we postulate that this sudden abdominal decompression could play a role in the postparacentesis systemic haemodynamic changes through mechanical decompression of the splanchnic vascular bed. A previous study showed that an acute increase in IAP, using mechanical compression, in cirrhotic patients caused a significant decrease in $\mathrm{CO}$ and an increase in MAP and SVR; the reverse occurred after releasing abdominal compression. These changes occur in the absence of variations in norepinephrine suggesting local myogenic vasoconstriction or mechanical compression of the splanchnic bed. ${ }^{16}$ Changes in external pressure on the body, such as those encountered in space flight or immersion in water, are associated with increased cardiac preload. ${ }^{17}$ Several lines of evidence have suggested that this effect is mediated by redistribution of blood volume with a relative increase in central blood volume. ${ }^{18}$ Previous
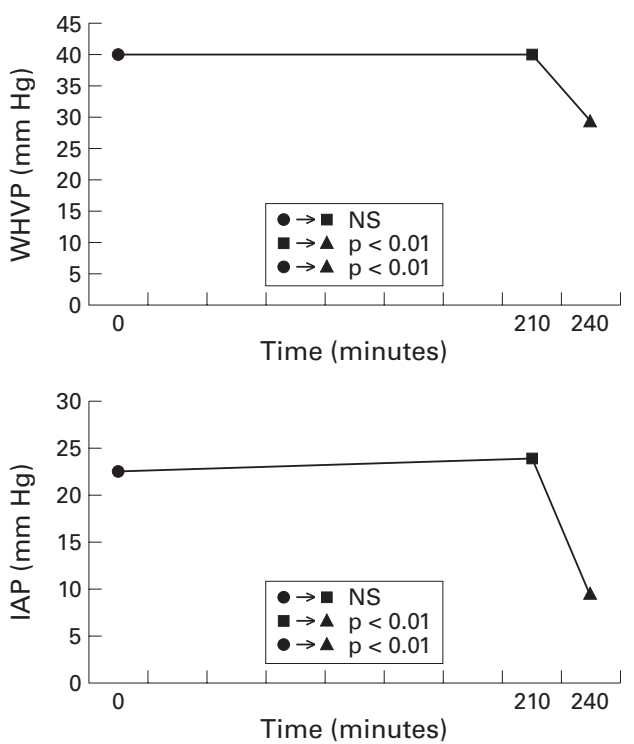

Figure 2 Splanchnic haemodynamic parameters at baseline, after completion of paracentesis, and after girdle deflation. FHVP, free hepatic vein pressure; WHVP, wedge hepatic vein pressure; HVPG, hepatic venous pressure gradient; IAP, intra-abdominal pressure. 
studies have shown that 60 minutes after a standard paracentesis, a significant increase in $\mathrm{CO}$ together with a reduction in MAP occur and consequently, calculated SVR experiences a significant decrease. ${ }^{2}{ }^{12} 19{ }^{20}$ In contrast, in our study, no significant haemodynamic changes were observed when IAP remained unchanged by compression of the pneumatic girdle. Interestingly, the characteristic haemodynamic pattern, previously described in patients undergoing standard paracentesis, was reproduced immediately after girdle deflation. In fact, calculated SVR was significantly decreased five minutes after girdle deflation.

The results of our study support the hypothesis that abrupt abdominal decompression, occurring immediately after tense ascites removal, could play an important role in the early postparacentesis systemic haemodynamic changes. It has been suggested that these early postparacentesis haemodynamic changes depend on an increase in cardiac preload after cava decompression and intrathoracic pressure reduction, ${ }^{321}{ }^{22}$ resulting in an increase in $\mathrm{CO}$ together with an initial decrease in PRA and plasma aldosterone concentration in some patients. The decrease in SVR in our study may be attributed in part to reflex inhibition of sympathetic vasoconstrictor tone secondary to an increase in CO. ${ }^{2}{ }^{3}$ However, Ruiz del Arbol et al showed that several days after paracentesis the decrease in SVR remained unchanged in spite of recovery of $\mathrm{CO}$ to baseline values. ${ }^{12}$ Therefore, this finding suggests that other mechanisms, independent of $\mathrm{CO}$, must be implicated in PCD observed in some patients days after massive ascites removal. A tentative explanation for this persistent decrease in SVR could be as follows. Ascites removal induces two opposing and simultaneous effects; one beneficial effect due to mechanical decompression of the inferior cava vein and reduction of thoracic pressure which improve $\mathrm{CO}$ and systemic haemodynamic conditions, and the other a deleterious effect due to mechanical decompression of an already dilated splanchnic vascular bed leading to further arterial vasodilatation in patients in whom the vascular response to the vasoactive system is blunted. ${ }^{23}$ Acute abdominal decompression could enhance a section of the arteriolar vessel by a direct mechanical effect or as a consequence of an adaptive regulation to compensate for the expanding capacity of the venous splanchnic area and its visceral vasculature produced after abdominal decompression, ${ }^{24}$ in a similar way that arteries proximal to a fistula dilate to compensate for an increase in flow rate. ${ }^{25}$ Beneficial haemodynamic effects derived from cava vein decompression initially hide the deleterious effects of abdominal decompression on the splanchnic vascular bed which only emerge when the former effect is exhausted. In spite of our hypothesis, we did not find a correlation between the magnitude of the decrease in IAP and that of SVR after abdominal decompression, but this does not exclude a role for abdominal decompression on the decrease in SVR. The lack of correlation between these two parameters in this small sample may be explained by different vascular reactivity to the effects of the vasoactive system.

Although we analysed the haemodynamic effects of paracentesis under constant IAP only up to one hour after paracentesis, the absence of postparacentesis haemodynamic changes while IAP remained constant and reproduction of typical haemodynamic changes just after girdle deflation suggests that IAP could play a role in the haemodynamic sequence that eventually leads to PCD in some patients.

We cannot definitively conclude from our data that a decrease in IAP is the cause of PCD appearing between 48 hours and six days after paracentesis, as described previously. ${ }^{7}$ However, indirect evidence would support this contention. Ginés and colleagues ${ }^{4}$ showed that the volume of ascites removed was predictive of the development of PCD. As IAP is a result of ascites volume and abdominal wall compliance, it must be taken into account as a possible pathogenic factor in PCD. A recent study showed that limb and abdominal compression in patients with cirrhosis improved renal function parameters and reduced activation of the renin-angiotensin system, suggesting that body compression increases effective circulating blood volume. ${ }^{26}$

Further studies need to determine if a controlled decrease in IAP after paracentesis, avoiding abrupt changes in systemic haemodynamics, can preclude the development of circulatory dysfunction and its clinical consequences.

This work was supported in part by grant No 97/0158 from the Ministerio de Sanidad y Consumo. The authors are indebted to Andrés T Blei MD and Luis Ruiz del Arbol MD for their criticism and advice. Presented in part at the 48th Meeting of the American Association for Study of Liver Diseases, Chicago, IL, USA, October 1997, and published as an abstract (Hepatology 1997;26:183A).

1 Ginés P, Titó LI, Arroyo V, et al. Randomized comparative study of therapeutic paracentesis with and without intravenous 38 album

2 Panos MZ, Moore K, Panayiotis V, et al. Single total paracentesis for tense ascites: Sequential hemodynamic changes and right atrial size. Hepatology 1990;11:662-7.

3 Pozzi M, Osculati G, Boari G, et al. Time course of circulatory and humoral effects of rapid total paracentesis in cirrhotic patients with tense ascites. Gastroenterology 1994; 106:709-19.

4 Ginés A, Fernández-Esparrach G, Monescillo A, et al. Randomized trial comparing albumin, dextran 70 , and polygeline in cirrhotic patients with ascites treated by paracentesis. Gastroenterology 1996;111:1002-10.

5 Peltekian KM, Wong F, Liu PP, et al. Cardiovascular, renal, and neurohumoral responses to single large volume paracentesis in patients with cirrhosis and diuretic-resistant ascites. Am f Gastroenterol 1997;92:394-9.

6 Planas R, Gines P, Arroyo V, et al. Dextran-70 versus albumin as plasma expander in cirrhotic patients with tense ascites treated with total paracentesis. Result of a randomized study. Gastroenterology 1990;99:1736-44.

7 Ginés P, Arroyo V, Quintero E, et al. Comparison of paracentesis and diuretics in the treatment of cirrhotics with tense ascites. Results of a randomized study. Gastroenterology 1987;93:234-41

8 Pinto PC, Amerian J, Reynolds TB. Large volume paracentesis in non-edematous patients with tense ascites: its effect on intravascular volume. Hepatology 1988;8:207-10.

9 Saló J, Ginés A, Ginés P, et al. Effect of therapeutic paracentesis on plasma volume and transvascular escape rate of albumin in patients with cirrhosis. F Hepatol 1997;27:64553.

10 Cabrera J, Inglada L, Quintero E, et al. Large-volume paracentesis and intravenous saline: effects on the reninangiotensin system. Hepatology 1991;14:1125-8.

11 Salerno F. Large-volume paracentesis and volume reexpansion: can synthetic plasma expanders safely replace
ale albumin? f Hepatol 1992;14:143-5.

12 Ruiz-Del-Arbol L, Monescillo A, Jiménez W, et al. Paracentesis-induced circulatory dysfunction: mechanism 
and effect on hepatic hemodynamics in cirrhosis. Gastroenterology 1997;113:579-86.

13 Knauer CM, Lowe HM. Hemodynamics in the cirrhotic patient during paracentesis. N Engl F Med 1967;276:491-6. 4 Vila MC, Solá R, Molina L, et al. Hemodynamic changes in patients developing effective hypovolemia after total paracentesis. F Hepatol 1998;28:639-45.

15 Kotelanski B, Groszmann R, Cohn JN. Circulation times in the splanchnic and hepatic beds in alcoholic liver disease. Gastroenterology 1972;63:102-11.

16 Luca A, Cirera I, García-Pagán JC, et al. Hemodynamic effects of acute changes in intraabdominal pressure in patients with cirrhosis. Gastroenterology 1993;104:222-7.

17 Schlant RC, Sonnenblick EH. Normal physiology of the cardiovascular system. In: Schlant RC, Alexandre RW, eds. The heart. Arteries and veins. New York: McGraw-Hill, 1994:113-51.

18 Epstein M. Cardiovascular and renal effects of head-out water immersion in man. Application of the model in the assessimmersion in man. Application of the model in the as
ment of volume homeostasis. Circ Res 1976;39:619-28.

19 Luca A, Feu F, Garcia-Pagan JC, et al. Favorable effects of total paracentesis on splanchnic hemodynamic in cirrhotic patients with tense ascites. Hepatology 1994;20:30-3.
20 Simon DM, McCain JR, Bonkovsky HL, et al. Effects of therapeutic paracentesis on systemic and hepatic hemodynamics and on the renal and hormonal function. Hepatology 1987;7:423-9

21 Guazzi M, Polese A, Magrini F, et al. Negative influences of scites on the cardiac function of cirrhotic patients. Am f Med 1975;59:165-70.

22 Ranninger K, Switz DM. Local obstruction of inferior vena cava by massive ascites. Am f Roentgenol 1965;93:935-9.

23 Castro A, Jiménez W, Claria J, et al. Impaired responsiveness to angiotensin II in experimental cirrhosis: role of nitric to angiotensin II in experimental

24 Olerud S. Experimental studies on portal circulation increase intraabdominal pressure. Acta Physiol Scand 1953; 30(suppl): 109-14.

25 Kamiya A, Togawa T. Adaptive regulation of wall shear stress to flow change in the canine carotid artery. $\mathrm{Am} \mathrm{f}$ Physiol 1980;239:H14

26 Uemura M, Matsumoto M, Tsujii T, et al. Effects of "body compression" on parameters related to ascites formation: compression" on parameters related to ascites formation:
therapeutic trial in cirrhotic patient. $f$ Gastroenterol therapeutic trial 